\title{
Anti-Inflammatory Therapeutic Development and Optimization of Umbilical Cord Tissue Derived Mesenchymal Stem Cells
}

\author{
Morgan T Sutton ${ }^{1}$, Sukhmani Kaur ${ }^{1,2}$, Katherine S Brown ${ }^{3}$, Matthew L Skiles ${ }^{3}$, Michael A Folz ${ }^{1}$, Arnold I Caplan ${ }^{4}$ and Tracey L Bonfield ${ }^{1^{*}}$ \\ ${ }^{1}$ Department of Pediatrics, Case Western Reserve University, Cleveland, Ohio, USA \\ ${ }^{2}$ Hathaway Brown School, Shaker Heights, Ohio, USA \\ ${ }^{3}$ CBR Systems Inc., South San Francisco, California, USA \\ ${ }^{4}$ Department of Biology, Skeletal Research Center, Case Western Reserve University, Cleveland Ohio, USA
}

"Corresponding author: Tracey L Bonfield, Department of Pediatrics, Allergy and Sleep Medicine, Case Western Reserve University, Cleveland, Ohio, USA, Tel: (216) 368-4558; Fax: (216) 368-4223; E-mail: Tracey.Bonfield@case.edu

Received date: July 30, 2018; Accepted date: August 22, 2018; Published date: August 27, 2018

Copyright: (c) 2018 Sutton MT, et al. This is an open-access article distributed under the terms of the Creative Commons Attribution License, which permits unrestricted use, distribution, and reproduction in any medium, provided the original author and source are credited.

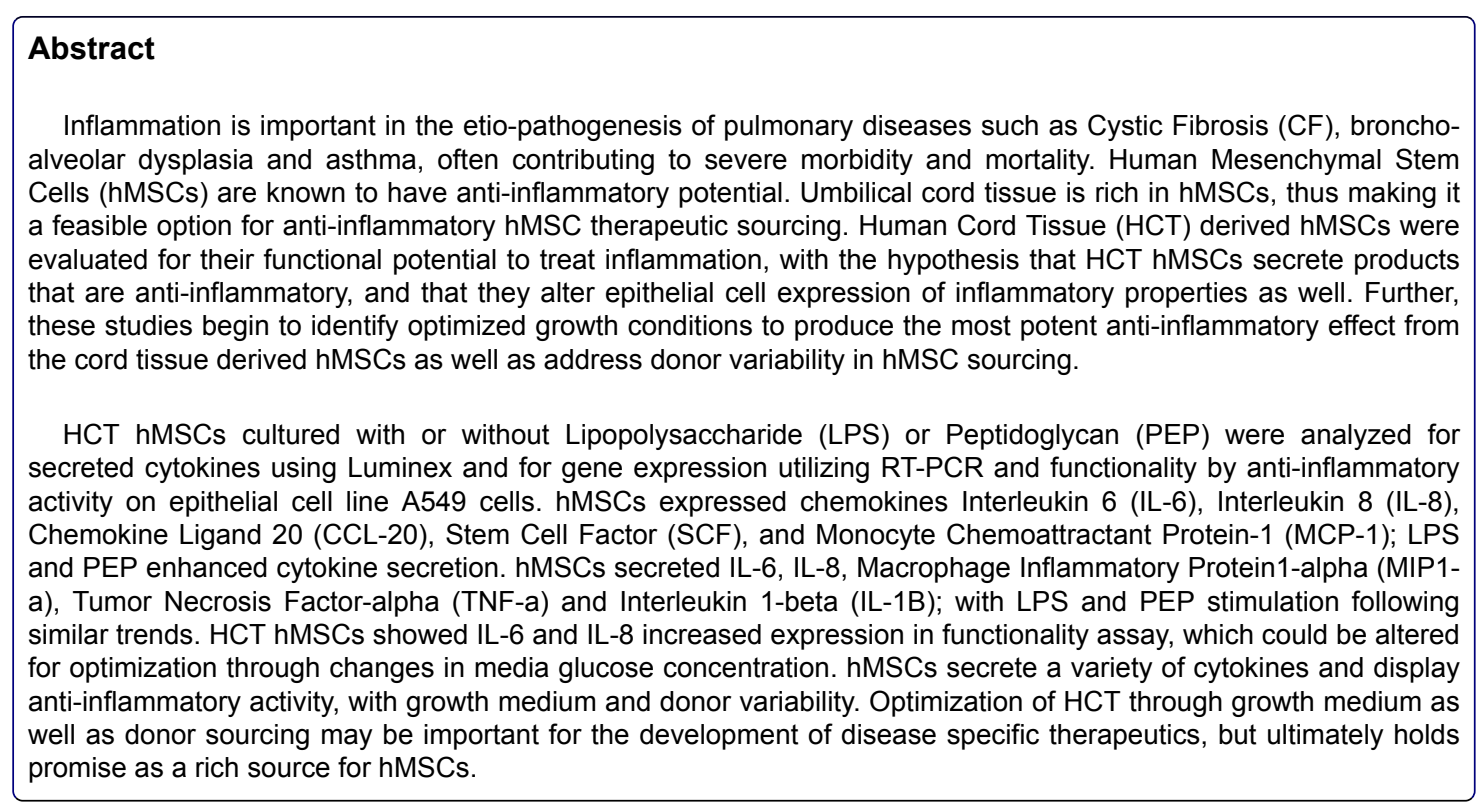

Keywords: Mesenchymal stem cells; Umbilical cord tissue; Antiinflammatory; Chemokines

Abbreviations: CF: Cystic Fibrosis; hMSCs: Human Mesenchymal Stem Cells; HCT: Human Cord Tissue; LPS: Lipopolysaccharide; PEP: Peptidoglycan; IL: Interleukin; BM: Bone Marrow; SCF: Stem Cell Factor; MCP: Monocyte Chemoattractant Protein; MIP: Macrophage Inflammatory Protein; TNF: Tumor Necrosis Factor; US: Unstimulated; Mes: MesenCult; LG: Low Glucose; HG: High Glucose

\section{Introduction}

Lung diseases, especially asthma and Chronic Obstructive Pulmonary Disease (COPD), are responsible for the death of approximately 4 million people yearly worldwide [1]. Air pollution, smoking, inflammation, and genetic mutations contribute to lung diseases by deteriorating lung capacity and impeding ventilation and the supply of adequate oxygen to the body. The symptoms of lung disease may include dyspnea, wheezing, cough, and chest pain with the underlying pathogenic mechanisms for most lung disease being inflammation and infection [2]. Severe inflammation and the inability to resolve it ultimately leads to build up of infectious pathogens, such as Pseudomonas aeruginosa, and can generate severe lung damage, resulting in pulmonary failure [3].

Human Umbilical Cord Blood (HUCB) has been previously utilized as a source of hematopoietic stem cells. These HUCB stem cells are multi-potent and have been shown to have regenerative, antiinflammatory, and bioactive properties [4]. hMSCs derived from cord blood and Bone Marrow (BM) are bioactive and can be used to ameliorate inflammation and to augment bactericidal capabilities in asthma and CF [5]. In the majority of clinical trials, hMSCs have been derived from BM aspirates of healthy volunteers, which is both an invasive and a costly procedure [6]. HCT has been investigated as a potential, rich source of hMSCs, as it is often discarded as human medical waste [7]. Further, with the availability and abundance of a source material, the non-invasive collection procedure, and the lack of ethical concerns that are associated with other sources, HCT are an attractive option for therapeutic hMSC sourcing.

In these studies, we profiled HCT hMSCs ability to produce and secrete anti-inflammatory products, ultimately pursuing the identification of the mechanistic response of HCT hMSCs through phenotypic properties in inflammatory scenarios, such as bacterial analog exposure. We further determined the functional capacity of 
HCT hMSCs by examining effects on cellular activity with airway epithelial cells. Finally, we present options for HCT growth optimization towards specific anti-inflammatory properties, and define donor variability as a factor to be considered when utilizing HCT hMSCs as a therapeutic source. The innovative nature of the results in this manuscript, as well as their alignment with our findings in BM derived hMSCs demonstrate the unique role hMSCs can play in terms of their therapeutic potential for chronic inflammatory diseases. Thus, HCT hMSCs may be considered as an economical, advantageous, and potent resource of stem cells for therapeutic development.

\section{Materials and Methods}

\section{Umbilical cord tissues}

Donated umbilical cord tissue was collected from consenting mothers, transported to a processing facility in Tucson, Arizona and cryopreserved as a composite material in a DMSO-based freeze media (CryoStor ${ }^{\circledR}$ CS10, BioLife Solutions, Bothell, WA). To isolate HCT hMSCs, the cryopreserved cord tissue was rapidly thawed and cells were isolated by explant outgrowth [8]. After expansion the cells were cryopreserved as single cell suspensions from individual donors and shipped overnight on dry ice to Case Western Reserve University.

\section{hMSC preparation and testing}

This study was performed at Case Western Reserve University, and was approved by Institutional Review Board Committees. HCT hMSCs in frozen $\left(-80^{\circ} \mathrm{C}\right)$ vials were obtained from Cord Blood Registry ${ }^{\circ}$ as prepared above, and were identified by donor number. The hMSCs were isolated and grown following the standard operating procedures using MesenCult (Mes) Medium (STEMCELL Technologies Inc. Cambridge MA) [9] and Low Glucose (LG) Dulbecco's Modified Eagle's medium (1 g/L; DMEM; Thermofisher; Waltham, MD) and High Glucose (HG) DMEM Media (4.5 g/L; Thermofisher; Waltham, $\mathrm{MD})$; methods for which clinical trial based hMSCs are maintained. $[10,11]$. hMSCs were cultured in the following environments: no stimulant $(20 \mu \mathrm{L} / 2 \mathrm{~mL}$ cord media), $20 \mu \mathrm{L} / 2 \mathrm{~mL}$ cord media with LPS $(10 \mu \mathrm{g} / \mathrm{mL}$; LPS; Sigma Aldrich; St. Louis, MO) and $20 \mu \mathrm{L} / \mathrm{mL}$ cord media with PEP (Sigma Aldrich; St. Louis MO). They were then incubated for 24 hours.

\section{A549 preparation and testing}

The A549 epithelial cells were grown in ATCC media in the presence of L-glutamine, with 5000 units/mL of both Penicillin (base) and Streptomycin to prevent bacterial contamination of the epithelial cells $[12,13]$, and were utilized at passage 8 or 9 . Cells were grown in 2 $\mathrm{mL}$ of A549 media. One day prior to harvest, $1 \mathrm{~mL}$ of cord tissue media was added in place of $1 \mathrm{~mL}$ of A549 media. Three hours later they were cultured with cord tissue media, followed by stimulation with or without LPS. Cells were harvested 24 hours post stimulation and cell pellets were saved for gene expression assays using Taqman Real Time PCR 7500 (ABI; Foster City, CA).

\section{HCT gene expression by Reverse Transcriptase-Polymerase Chain Reaction (RT-PCR)}

hMSCs were processed for messenger Ribonucleic Acids (mRNA) followed by complementary Deoxyribonucleic Acid (cDNA) synthesis for chemokine gene expression using RT-PCR. Quality of mRNA and
cDNA was assessed through Nanodrop spectrophotometry (optimal threshold $260-280 \mathrm{~nm}$ ). Validation was done through use of a reference gene Peptidyl Prolyl Isomerase (hPPIA). The expression of IL-6, IL-8, MIP3- $\alpha$ (CCL20), SCF and chemokine ligand 2 (MCP-1) was compared to the expression of hPPIA (all primers, and extraction reagents from ABI; Foster City CA). All PCR samples were compared to PPIA expression for fold changes in each target gene threshold cycle (dCT) value. This was normalized to baseline control to measure overall response to stimulations, as previously described (ddCT) [14].

\section{HCT secreted products by luminex assay}

hMSC supernatants were analyzed for secreted cytokines using a Luminex assay at baseline (Unstimulated (US)) and after treatment with LPS and PEP. The levels of cytokine IL-6, IL-8, MIP1- $\alpha$, TNF- $\alpha$ and IL1- $\beta$ were measured. Supernatants from samples $(20 \mu \mathrm{L})$ were interfaced with luminescence beads with ratios of proprietary fluorophores. Each bead is specific for a cytokine or chemokine. As the sample interacts with the beads, they capture antibodies conjugated to the beads and bind to the cytokine/chemokine which is followed by a second antibody labeled with Phycoerythrin (PE). The detection of the cytokine/chemokine is based upon detection of the unique bead ID and the detection of the PE using a dual laser system with a lower end sensitivity of $3.2 \mathrm{pg} / \mathrm{ml}[15,16]$. Each cytokine/chemokine is compared to its specific standard curve, also done in multiplex (R\&D Systems Inc.; Minneapolis MN). Data is expressed as mean $(\mathrm{pg} / \mathrm{ml} \pm \mathrm{SEM}$, $n=3$ ).

\section{HCT functionality assays}

To monitor the effect of hMSCs on the A549 airway epithelial cells, epithelial cells were stimulated with cord media (1 mL Mes, LG DMEM and HG DMEM and $1 \mathrm{~mL}$ epithelial cell media), cord media with LPS $(20 \mu \mathrm{LPS} / 2 \mathrm{~mL} ; 1 \mathrm{~mL}$ cord media and $1 \mathrm{~mL}$ epithelial cell media), cord stem cells $(1 \mathrm{~mL}$ cord stem supernatant with $1 \mathrm{~mL}$ epithelial cell media), and cord stem cells with LPS $(20 \mu \mathrm{LPS} / 2 \mathrm{~mL} ; 1$ $\mathrm{mL}$ cord stem cell supernatant and $1 \mathrm{~mL}$ epithelial cell media). Cells were studied to determine if the cord tissue stem cells had an antiinflammatory effect against the epithelial cells and LPS. Inflammation was quantified by changes in IL- 8 and IL- 6 chemokine expression with RT-PCR.

\section{Statistical analysis}

Data in this manuscript is expressed as mean \pm Standard Error of Mean (SEM) using non-parametric Mann-Whitney Tests. Linear correlations and variance analysis were carried out using GraphPad Prism 6 (La Jolla, CA). Level of significance was determined by Student's t-tests using a two-way analysis with $\mathrm{P} \leq 0.05$ level of stringency. $\mathrm{P}$ values that are close to confidence interval $(5 \%)$ are provided in order to depict trends towards significance among the data. Different donors are shown as replicates. In cases where data was not able to be transformed by normality, use of non-parametric van Elteren tests (Van Elteren, 1960) compared groups. Significance between experimental groups and conditions is denoted by comparison lines. Stars above columns denote significance with $\mathrm{P}<0.005$. The Statistical Resource Center; Department of Pediatrics; Case Western Reserve University was utilized for these analyses. 
Citation: Sutton MT, Kaur S, Brown KS, Skiles ML, Folz MA, et al. (2018) Anti-Inflammatory Therapeutic Development and Optimization of Umbilical Cord Tissue Derived Mesenchymal Stem Cells. J Stem Cell Res Ther 8: 435. doi:10.4172/2157-7633.1000435

Page 3 of 9

\section{Results}

\section{Gene expression and cytokine secretion of HCT}

hMSCs cells from three donors were treated with and without LPS and PEP. LPS mimics the effect of gram-negative bacteria and is a component of the membrane that incites a strong immunological response. PEP is a bacterial analog made of amino acids and sugars that is found in the cell wall of all bacteria and in high concentrations in gram-positive bacteria. hMSCs were evaluated for IL-6, IL-8, MCP-1, CCL20 and SCF gene expression using RT-PCR. The Luminex multiplex assay was used to analyze the cytokines IL-6, IL-8, MIP1- $\alpha$, TNF- $\alpha$ and IL- $1 \beta$ from the three different donors in the secreted product of the cells. HCT hMSCs were first assayed to assess constitutive secretion of cytokines without stimulation, followed by the assessment of the response of HCT hMSCs to stimulation by LPS and PEP.

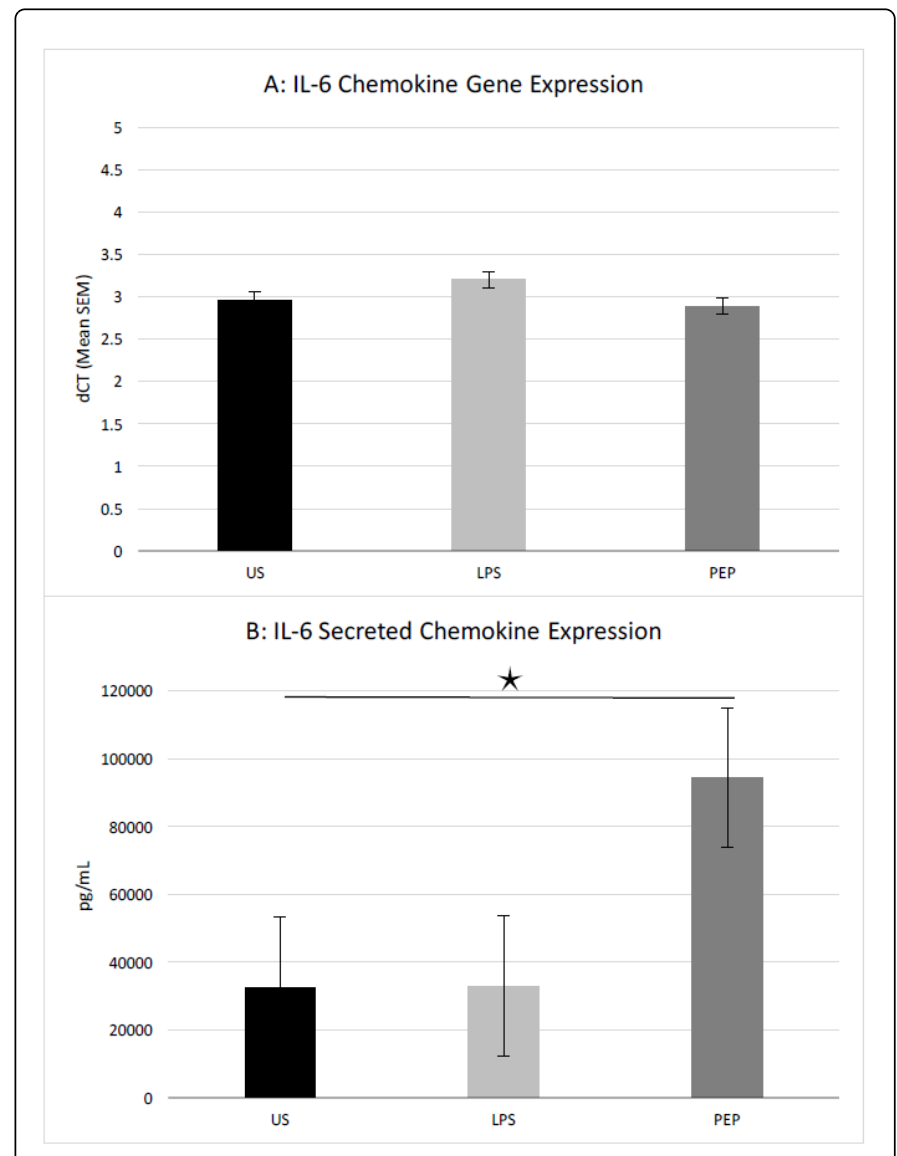

Figure 1: IL-6 expression in HCT hMSCs. A) HCT hMSC baseline gene expression of anti-inflammatory chemokine IL-6 for US, LPS and PEP conditions as compared to ubiquitously expressed housekeeping gene hPPIA ( $\mathrm{n}=3$; dCT (Mean SEM)). dCT is an indication of how long it takes for a gene to be expressed. B) Secreted expression of IL- 6 chemokine from HCT hMSCs for US, LPS and PEP conditions as compared to baseline expression levels $(\mathrm{n}=3 ; \mathrm{pg} / \mathrm{mL})$.

\section{HCT IL-6 production}

IL-6 is a known acute phase protein that induces multiple chemokines, including IL-10. It also exudes extensive antiinflammatory properties that could be beneficial in scenarios of chronic infection and inflammation [17]. HCT hMSCs that were US cells expressed IL- 6 at $2.958 \pm 0.386$ difference in cycle value compared to hPPIA; while LPS-stimulated HCT hMSCs expressed IL-6 at a difference of $3.200 \pm 1.070$ cycles and PEP-stimulated HCT hMSCs expressed IL-6 at a difference of $2.883 \pm 0.444$ cycles $(n=3)$ (Figure 1A). These values suggest the constitutive expression on IL- 6 by HCT hMSCs at a high level. Further, gram-positive bacterial stimulation decreased the threshold cycling time difference, suggesting a trend towards increased anti-inflammatory cytokine expression in scenarios of bacterial exposure that lead to an influx of inflammation $(n=3$; $\mathrm{P}=0.835 ; \mathrm{t}=-0.223$ ) (Figure $1 \mathrm{~A}$; Columns 2 and 3 ). This trend is consistent in the findings of the Luminex secreted data as well (Figure 1B). US HCT hMSCs showed IL-6 secretion of 32,620.949 $\pm 13,991.013$ $\mathrm{pg} / \mathrm{mL}$ over baseline, compared to $94,563.838 \pm 11,685.220 \mathrm{pg} / \mathrm{mL}$ with PEP stimulation, demonstrating statistical significance of the findings $(\mathrm{n}=3 ; \mathrm{P}=0.004 ; \mathrm{t}=5.885)$ (Figure 1B; Column 3). LPS stimulation showed secretion levels of $32969.982 \pm 9114.093 \mathrm{pg} / \mathrm{mL}$ similar to what was seen in US HCT hMSCs (Figure 1B; Column 2).

\section{HCT IL-8 production}

IL-8 is a protein that causes chemotaxis in target cells, ultimately shifting the cell differential towards an acute neutrophil influx, especially in scenarios of severe infection, making them proinflammatory [17]. This pro-inflammatory response may be harmful or beneficial in scenarios of pulmonary disease, depending on ability to ameliorate chronic infection. HCT hMSCs that were US cells expressed IL-8 at $3.887 \pm 3.000$ difference in cycle value more than hPPIA; while LPS stimulated HCT hMSCs expressed IL-8 at a difference of $3.811 \pm$ 2.907 cycles and PEP stimulated HCT hMSCs expressed IL-8 at a difference of $4.146 \pm 3.443$ cycles over hPPIA $(n=3)$ (Figure 2A).

In the supernatants, US HCT hMSCs showed IL-8 secretion of $93,146.169 \pm 9312.900 \mathrm{pg} / \mathrm{mL}$ above baseline, compared to $139,275.003$ $\pm 9,492.796 \mathrm{pg} / \mathrm{mL}$ with PEP stimulation, demonstrating statistical significance of the findings $(\mathrm{n}=3 ; \mathrm{P}=0.004 ; \mathrm{t}=6.008)$ (Figure 2B; Column 3). LPS stimulation showed secretion levels of $84,177.502 \pm$ $2,919.594 \mathrm{pg} / \mathrm{mL}$ (Figure 2B; Column 2). These findings suggest that IL-8 is expressed by HCT hMSCs and their secreted products, and that IL- 8 expression can be altered by gram positive and gram negative bacterial analog exposure.

\section{HCT hMSCs express other anti-inflammatory genes}

CCL20 is chemotactic for both dendritic cells and lymphocytes, and also has the capability to enhance neutrophil recruitment, though to a much lesser extent. CCL20 may also be involved in attenuating cellular microbial response, making it a chemokine of significance for study. It should be noted that CCL20 expression is dependent on IL-6 expression, which we have presented above. HCT hMSCs that were US cells expressed CCL20 at $-4.385 \pm 3.465$ difference in cycle value compared to hPPIA; while LPS-stimulated HCT hMSCs expressed CCL-20 at a difference of $-4.907 \pm 2.107$ cycles and PEP-stimulated HCT hMSCs expressed CCL20 at a difference of $-4.472 \pm 2.371$ cycles $(n=3)$ (Figure 3; First Column Set). This data suggest a lessened production of CCL20 by HCT hMSCs, which is further downregulated by bacterial analog exposure $(-4.385 \pm 3.465$ versus: gram negative 
Citation: Sutton MT, Kaur S, Brown KS, Skiles ML, Folz MA, et al. (2018) Anti-Inflammatory Therapeutic Development and Optimization of Umbilical Cord Tissue Derived Mesenchymal Stem Cells. J Stem Cell Res Ther 8: 435. doi:10.4172/2157-7633.1000435

Page 4 of 9

$-4.907 \pm 2.107$; gram positive $-4.472 \pm 2.371$ ) (Figure 3; First Column Set; Columns 2 and 3).

SCF is an autologous growth factor for stem cell growth and differentiation, enhancing the dynamic nature of the cells. Further, it has been suggested that SCF may increase the potency of hMSCs in contributing to their environment. HCT hMSCs that were US expressed SCF at $7.603 \pm 1.533$ differences in cycle value compared to hPPIA ubiquitous expression. LPS-stimulated HCT hMSCs expressed SCF at a difference of $7.557 \pm 2.497$ cycles and PEP-stimulated HCT hMSCs expressed SCF at a difference of $7.689 \pm 2.649$ cycles compared to hPPIA ( $n=3)$ (Figure 3; Second Column Set). This high expression of SCF is suggestive of the ability of HCT hMSCs to contribute actively to their surroundings, both on their own and in the context of bacterial exposure.
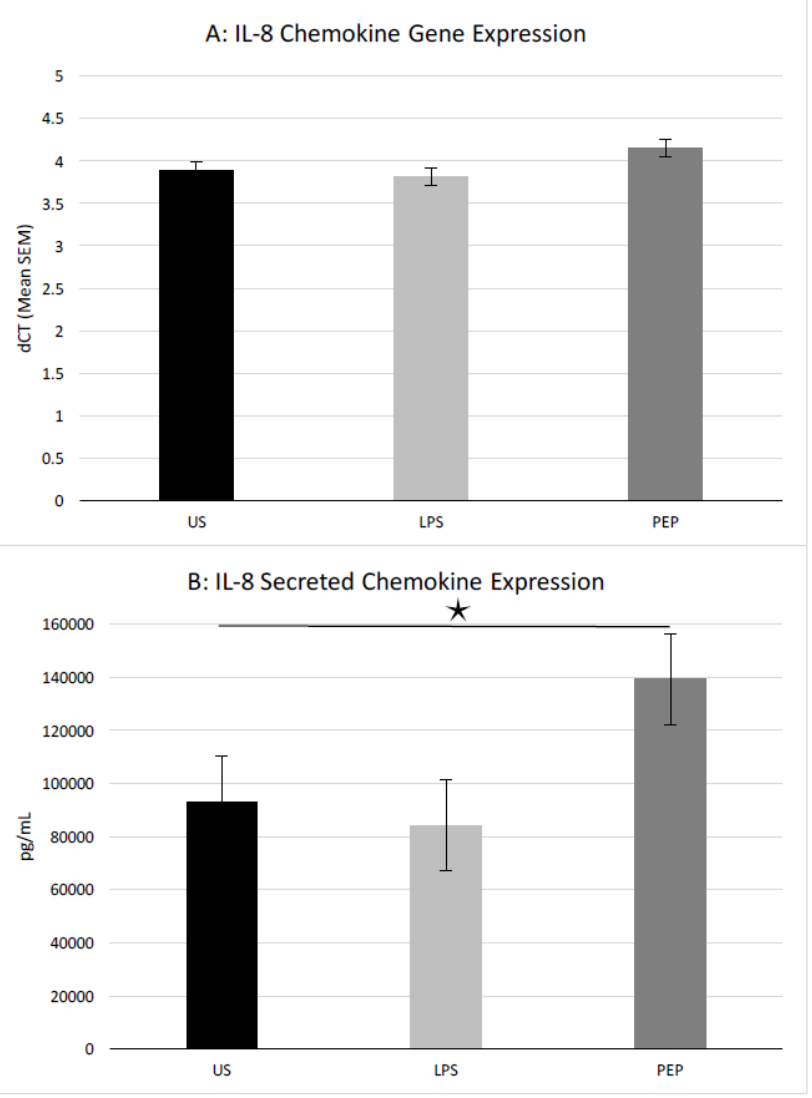

Figure 2: IL-8 expression in HCT hMSCs. A) HCT hMSC baseline gene expression of inflammatory chemokine IL-8 for US, LPS and PEP conditions as compared to ubiquitously expressed housekeeping gene hPPIA ( $\mathrm{n}=3$; dCT (Mean SEM)). dCT is an indication of how long it takes for a gene to be expressed. B) Secreted expression of IL-8 chemokine from HCT hMSCs for US, LPS and PEP conditions as compared to baseline expression levels $(\mathrm{n}=3 ; \mathrm{pg} / \mathrm{mL})$.

MCP-1 is a chemokine that is directly involved in monocyte and helper-t-cell recruitment; both types of cells are needed in scenarios of chronic inflammation [3]. MCP-1 expression was increased in US HCT hMSCs as opposed to hPPIA gene expression by $2.581 \pm 1.220$ difference in cycle value. LPS-stimulated HCT hMSCs expressed MCP- 1 at a difference of $2.781 \pm 1.909$ cycles and PEP-stimulated HCT hMSCs expressed MCP- 1 at a difference of $2.408 \pm 1.724$ cycles over hPPIA ( $n=3$ ) (Figure 3; Column Set 3). MCP-1 seems to be highly expressed by HCT hMSCs both in the presence and absence of bacterial analogs, which may alter its expression of the monocyte recruiter.

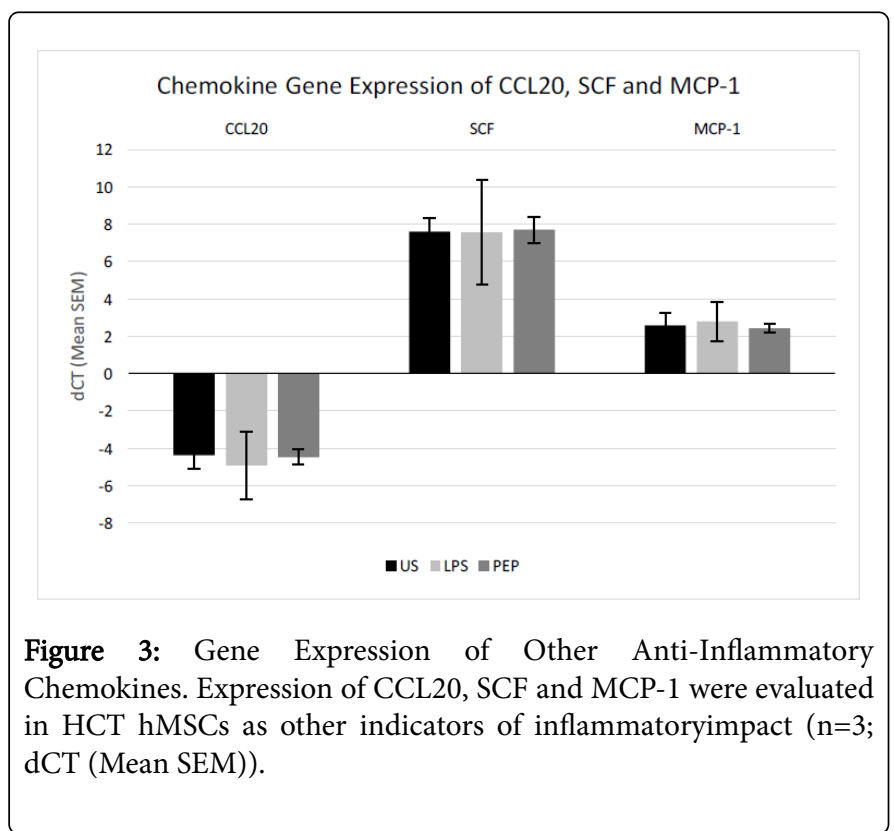

\section{HCT hMSCs secrete other anti-inflammatory chemokines}

US HCT hMSCs showed secretion of MIP1- $\alpha$, a major macrophage chemokine at $1,990.366 \pm 657.6 \mathrm{pg} / \mathrm{mL}$ above baseline. This expression was enhanced with both PEP stimulation $(2,919.500 \pm 460.8167$ $\mathrm{pg} / \mathrm{mL} ; \mathrm{n}=3 ; \mathrm{P}=0.116 ; \mathrm{t}=2.004$ compared to US) (Figure 4; First Column Set; Column 3) and significantly with LPS stimulation, $(9,312.901 \pm 607.817 \mathrm{pg} / \mathrm{mL} ; \mathrm{n}=3 ; \mathrm{P}=0.0001 ; \mathrm{t}=14.163$ compared to US) (Figure 4; First Column Set; Column 2). MIP1- $\alpha$ is imminent for immune response towards infection and inflammation.

US HCT hMSCs showed TNF- $\alpha$ secretion, a pro-inflammatory chemokine involved in systemic inflammation at $936.366 \pm 224.500$ $\mathrm{pg} / \mathrm{mL}$ above baseline (Figure 4; Second Column Set). This expression was also seen with PEP stimulation $(1,007.600 \pm 60.816 \mathrm{pg} / \mathrm{mL} ; \mathrm{n}=3$; $\mathrm{P}=0.623$; $\mathrm{t}=0.530$ compared with US) (Figure 4; Second Column Set; Column 3) and was slightly lower with LPS stimulation, (898.700 \pm 187.765 pg/mL; n=3) (Figure 4; Second Column Set; Column 2).

US HCT hMSCs showed IL- $1 \beta$ secretion, a pro-inflammatory, lymphocyte activating chemokine at 2,615.784 $\pm 171.885 \mathrm{pg} / \mathrm{mL}$ above baseline (Figure 4; Third Column Set). This expression was enhanced with PEP stimulation $(3,421.964 \pm 160.716 \mathrm{pg} / \mathrm{mL} ; \mathrm{n}=3 ; \mathrm{P}=0.004$; $\mathrm{t}=5.934$ compared with US) (Figure 4; Third Column Set; Column 3) and was similar with LPS stimulation $(2,600.176 \pm 245.876 \mathrm{pg} / \mathrm{mL}$; $\mathrm{n}=3$ ) (Figure 4; Third Column Set; Column 2), suggesting that bacterial exposure may enhance pro-inflammatory cytokine secretion. 
Citation: Sutton MT, Kaur S, Brown KS, Skiles ML, Folz MA, et al. (2018) Anti-Inflammatory Therapeutic Development and Optimization of Umbilical Cord Tissue Derived Mesenchymal Stem Cells. J Stem Cell Res Ther 8: 435. doi:10.4172/2157-7633.1000435

Page 5 of 9

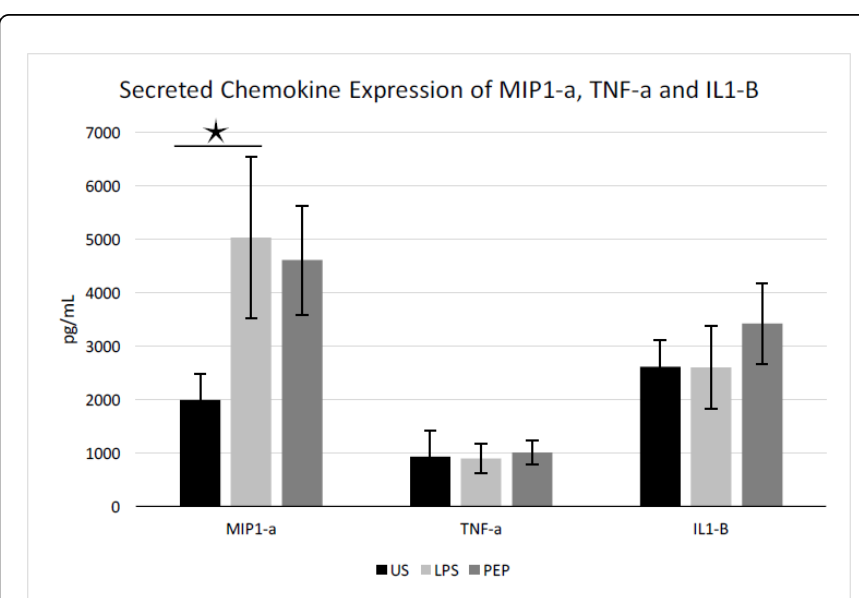

Figure 4: Secreted expression of other anti-inflammatory cytokines. Secreted expression of MIP1- $\alpha$, TNF- $\alpha$, and IL1- $\beta$, all important indicators of inflammatory response, were evaluated by Luminex for secretion from HCT hMSCs $(\mathrm{n}=3 ; \mathrm{pg} / \mathrm{mL})$.

\section{Anti-inflammatory functionality assays}

A549 airway epithelial cells produce IL-6 in their US state (12.217 \pm 1.992 cycles above hPPIA), but this production is decreased by gram negative bacterial analog stimulation (LPS: $14.113 \pm 1.557$ cycles), with the number of cycles 1.896 times longer for LPS $(n=3 ; P=0.264$; $\mathrm{t}=1.299$ ) (Figure 5A; Column 1). Treatment with HCT hMSCs significantly increased baseline expression of IL-6 by $6.573 \pm 3.661$ cycles $(\mathrm{n}=3 ; \mathrm{P}=0.012 ; \mathrm{t}=-4.354)$ (Figure $5 \mathrm{~A}$; Column 2). This same trend followed for LPS-stimulated A549 treated with HCT hMSCs, with a significant increase in expression by $7.552 \pm 0.837$ cycles $(n=3$; $\mathrm{P}=0.004 ; \mathrm{t}=-5.898$ ) (Figure 5A; Column 3). These trends clearly depict the capability of HCT to generate production of anti-inflammatory cytokine IL-6, both in the presence and absence of gram negative bacterial exposure. A549 airway epithelial cells express IL-8 in their US state $(8.450 \pm 0.891$ cycles above hPPIA $)$, which is not significantly altered by LPS stimulation $(8.528 \pm 1.922$ cycles; $\mathrm{n}=3 ; \mathrm{P}=0.954$; $\mathrm{t}=0.062$ ) (Figure 5B; Column 1). However, the addition of HCT hMSCs to the A549 showed a significant increase in the IL-8 expression, significantly decreasing the cycle number difference by $5.877 \pm 2.377$ cycles $(n=3 ; P=0.004 ; t=5.840)$ (Figure 5B; Column 2). Following this pattern, the LPS-stimulated A549s treated with HCT hMSCs showed an increase in IL-8 expression with an almost significant decrease in cycle number difference by $5.900 \pm 0.779$ cycles $(n=3 ; P=0.051$; $\mathrm{t}=-2.756$ ) (Figure 5B; Column 3). This suggests that HCT hMSCs may contribute to increased IL-8 production both in an infected and noninfected state for airway epithelial cells, a finding that could suggest both a therapeutic potential and concern, depending on the patient population.

\section{Optimization of growth of cord tissue stem cells}

In order to determine if anti-inflammatory chemokine secretion could be fine-tuned for therapeutic optimization, we utilized different concentrations of glucose DMEM in the growth medium (HG, LG, normal concentration Mes=MesenCult) to possibly create variations.

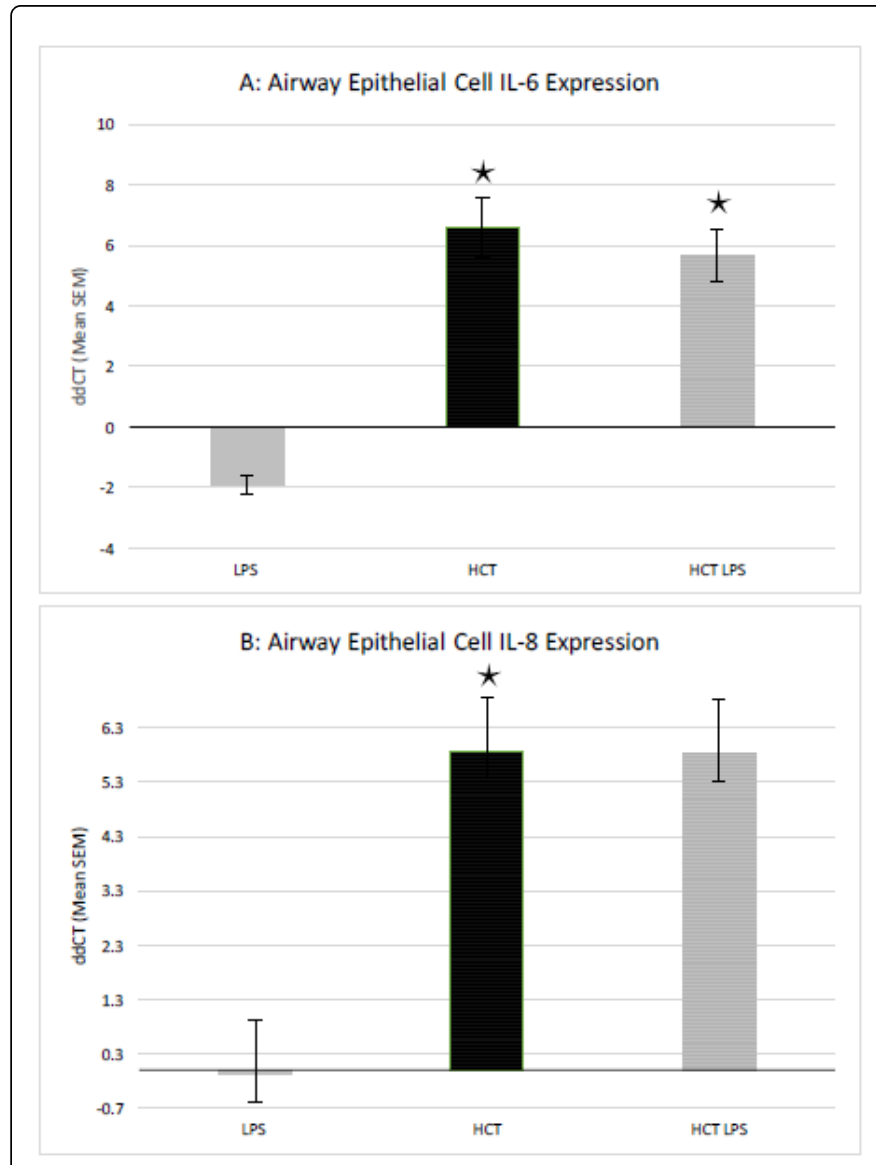

Figure 5: Anti-inflammatory functional impact in airway epithelial cells. A) Impact of HCT hMSCs on A549 airway epithelial cell IL-6 expression was evaluated for functionality implications $(n=3$; ddCT (Mean SEM)). ddCT represents the number of cycle differences between the US and each condition, indicating overall shift in inflammatory response. IL- 6 expression is increased by HCT hMSC exposure. B) Impact of HCT hMSCs on A549 airway epithelial cell IL-8 expression was evaluated for functionality implications $(n=3$; ddCT (Mean SEM)). ddCT represents the number of cycle differences between the US and each condition, indicating overall shift in inflammatory response. IL- 8 expression is increased by HCT hMSC exposure.

\section{IL-6 expression in optimization studies}

IL-6 was highly expressed for A549 in all media types, with no significant difference $(\mathrm{HG}=8.784 \pm 1.432 ; \mathrm{LG}=8.353 \pm 2.430$; Mes $=8.225 \pm 1.563)$ (Figure 6A). In all three media types, HCT hMSCs increased IL- 6 expression, with Mes media showing the largest increase $(2.955 \pm 0.878$ cycles shorter) (Figure 6A; Column 2). This increase was not significantly better than the HG media at $2.539 \pm 0.786(n=3$; $\mathrm{P}=0.574 ; \mathrm{t}=-0.611$ ) (Figure $6 \mathrm{~A}$; Column 3 ) or LG media at $1.970 \pm$ $0.765(\mathrm{n}=3 ; \mathrm{P}=0.217 ; \mathrm{t}=-1.465)$ (Figure $6 \mathrm{~A} ;$ Column 1). LPS stimulation did not significantly alter IL-6 expression in any media type $(\mathrm{HG}=8.655 \pm 1.542 ; \mathrm{LG}=8.956 \pm 1.330 ;$ Mes $=8.453 \pm 1.643)$ (Figure 6B; First Column Set). The response of LPS-stimulated A549 to HCT hMSCs in the varied medias showed a similar trend of increase in IL-6 expression to the baseline A549 cells, with increases in cycle 
Citation: Sutton MT, Kaur S, Brown KS, Skiles ML, Folz MA, et al. (2018) Anti-Inflammatory Therapeutic Development and Optimization of Umbilical Cord Tissue Derived Mesenchymal Stem Cells. J Stem Cell Res Ther 8: 435. doi:10.4172/2157-7633.1000435

Page 6 of 9

numbers of $(\mathrm{HG}=2.195 \pm 0.982 ; \mathrm{LG}=2.282 \pm 0.984 ;$ Mes $=1.279 \pm$ 1.769) (Figure 6B; Third Column Set). Though it had the largest increase in expression, LG media was not significantly better at increasing IL-6 expression than HG $(\mathrm{n}=3 ; \mathrm{P}=0.919 ; \mathrm{t}=-0.108)$ or Mes media $(\mathrm{n}=3 ; \mathrm{P}=0.439 ; \mathrm{t}=-0.858)$.

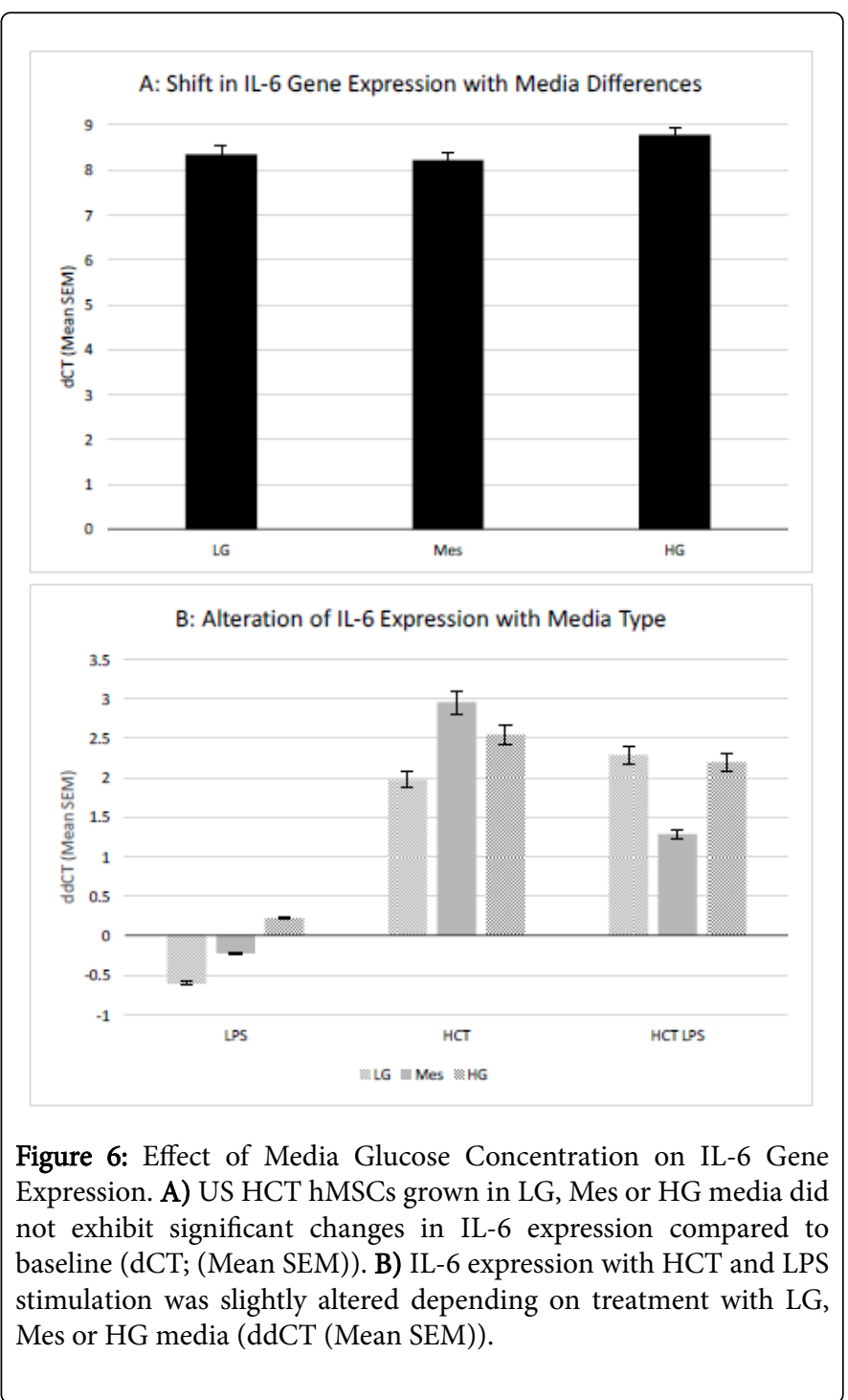

\section{IL-8 expression in optimization studies}

IL-8 was also expressed for all media types $(\mathrm{HG}=10.510 \pm 1.328$; $\mathrm{LG}=9.099 \pm 1.300$; Mes=7.569 \pm 1.093 ) (Figure 7A); however, it was expressed the least by Mes grown A549, a significant difference from the HG grown A549 $(n=3 ; P=0.0415 ; t=2.962)$ (Figure 7A; Columns 2 and 3 ), and large, but not statistically significant difference from the LG media grown A549 cells $(n=3 ; P=0.1937 ; t=1.560)$. Once again, in all three media types, HCT hMSCs increased IL- 8 expression, with HG media showing the largest increase $(3.541 \pm 0.843$ cycles) (Figure $7 \mathrm{~B}$; Second Column Set; Column 3). This increase was not significantly more than Mes media at $3.500 \pm 0.436(n=3 ; P=0.945 ; t=-0.073)$ (Figure 7B; Second Column Set; Column 2). However, the increase in IL-8 expression was significantly more for HG than LG media at 1.798 $\pm 0.135(\mathrm{n}=3 ; \mathrm{P}=0.024 ; \mathrm{t}=-3.534)$ (Figure 7B; Second Column Set; Columns 1 and 3$)$ and for Mes media compared to LG media ( $n=3$;
$\mathrm{P}=0.003 ; \mathrm{t}=-6.459$ ) (Figure 7B; Second Column Set; Columns 1 and 2) suggesting that the addition of HCT hMSCs may alter IL-8 expression differently depending on the glucose concentration of the media.

LPS stimulation of the A549 cells did not significantly alter IL-8 expression in any media type $(\mathrm{HG}=9.078 \pm 1.142$; $\mathrm{LG}=9.078 \pm 1.030$; Mes $=8.014 \pm 1.143$ ) (Figure 7A; First Column Set). When LPSstimulated A549 cells were treated with HCT hMSCs, IL-8 expression increased in all media types $(\mathrm{HG}=2.931 \pm 0.452 ; \mathrm{LG}=1.832 \pm 0.184$; Mes $=2.217 \pm 0.279$ ) (Figure 7B; Third Column Set). HG increased this expression significantly more than LG $(n=3 ; P=0.018 ; t=-3.901)$ (Figure 7B; Third Column Set; Columns 1 and 3) and trended towards significant increase over Mes media, as well $(\mathrm{n}=3 ; \mathrm{P}=0.080 ; \mathrm{t}=-2.328)$ (Figure 7B; Third Column Set; Columns 1 and 2). Conversely, LG was significantly better at reducing IL-8 expression over $\mathrm{HG}(\mathrm{n}=3 ; \mathrm{P}=0.018$; $\mathrm{t}=3.901$ ) (Figure 7B; First Column Set; Columns 1 and 3), and trended towards, though was not significantly better at, decreasing IL-8 expression with LPS stimulation as opposed to Mes media $(n=3$; $\mathrm{P}=0.117 ; \mathrm{t}=1.995$ ) (Figure 7B; First Column Set; Columns 1 and 2). These findings suggest that LG or Mes media may be optimal over HG media for decreasing IL-8 expression in response to stimulations.

\section{Discussion}

BM derived hMSCs have been found to be multipotent and regenerative, with angiogenic, anti-inflammatory, immunomodulatory, and anti-microbial properties [18]. BM hMSCs are therefore being investigated in the therapy of diseases associated with inflammation such as asthma and CF. In addition, the anti-microbial properties of BM hMSCs can be utilized to decrease the dose and frequency of antibiotics given in diseases where bacterial infections play a role in the morbidity related to the disease spectrum [19]. However, obtaining BM hMSCs is both invasive and costly. Despite the widespread use of $\mathrm{BM}$ in clinical trials, these factors highlight the importance of identifying alternative sources. The yield of MSCs from cord tissue is much higher and cord tissue collection is non-invasive and lacks the clinical costs associated with a BM extraction.

Cytokines are important to define the inflammatory response, to examine leukocyte recruitment, and to determine the type of cells that are involved in the immune response. The hMSCs were grown in the absence of any stimulants as well as in the presence of the LPS and PEP. It was anticipated that these stimulants would result in an increase in the inflammatory cytokines TNF $\alpha$ and IL- 6 in vitro, as noted in the B6.129S6-Cftr ${ }^{\text {tm2Uth }}(\mathrm{R} 117 \mathrm{H} / \mathrm{R} 117 \mathrm{H}$ mutation) and C57 BL/6 J controls (WT) mouse model. Cftrtm2Uth mice are a type IV Cftr mutant mice in which the effect of cellular therapies on the pulmonary effects of CF can be evaluated [4]. BM Macrophages from these models were stimulated with LPS, which increased mRNA expression of both IL-6 and TNF- $\alpha$ [4].

The Luminex assay done under different stimulant conditions showed that HCT hMSCs constitutively secreted IL- 6 and IL-8. The effect of the stimulants was to increase the production of IL-6 and IL-8 compared to the US secretion of these products. While it might appear paradoxical that hMSCs secrete cytokines that have inflammatory effects under the influence of stimulants that mimic bacterial infection, it is important to remember that hMSCs have been previously shown to produce cytokines and growth factors in the presence of tissue injury and inflammation [20]. This property of hMSCs highlights their capability to be responsive to the environment in which these stem cells reside in vivo [20]. It has been previously shown in mouse models 
that introduction of BM hMSCs caused a significant decline in the number of neutrophils and eosinophils in their environment. Histologically there was a decline in airway inflammation and also in the amount of epithelial cell lining thickening [18].

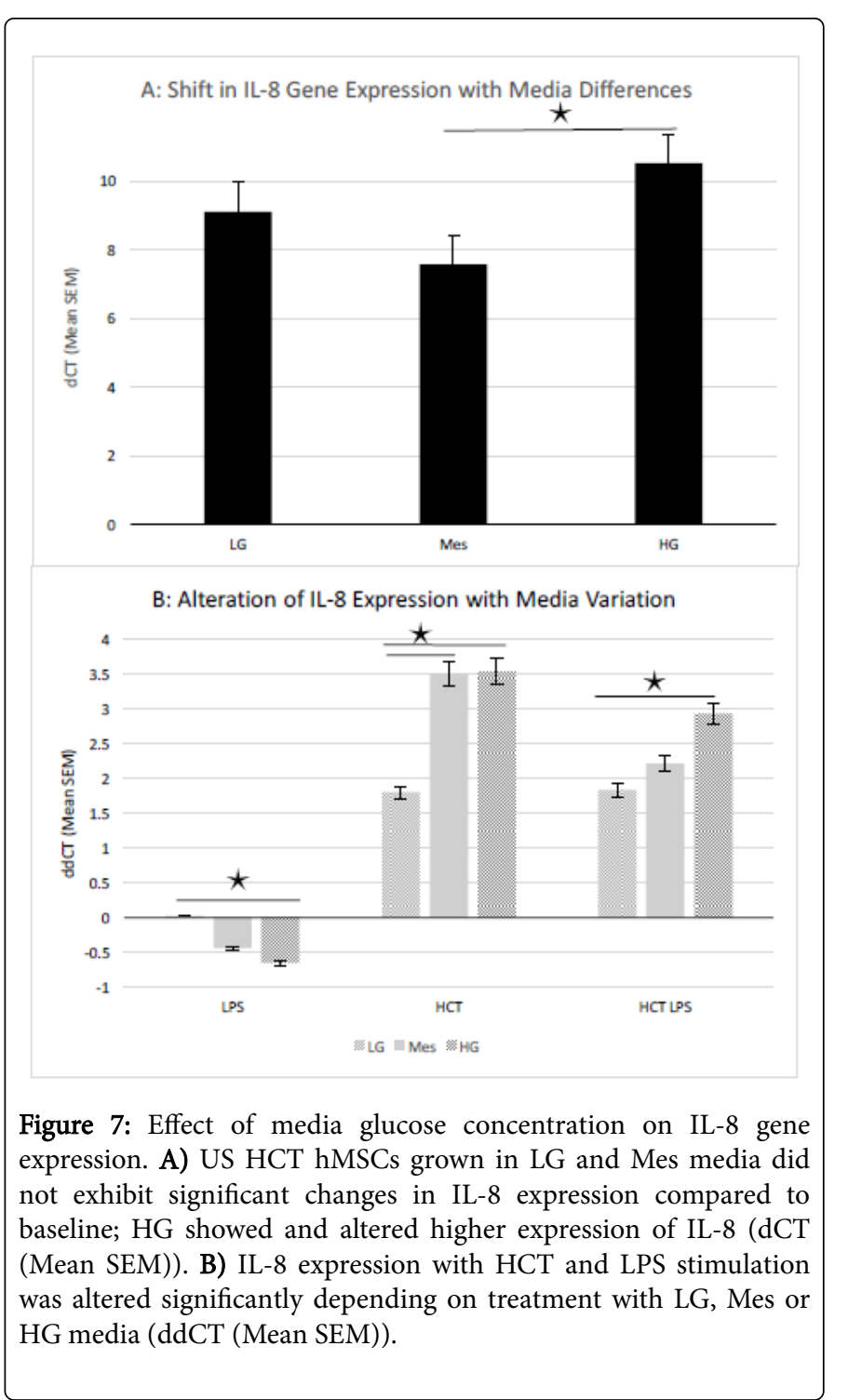

To investigate if the enhanced secretion of these cytokines correlated with increase in gene expression, we used the Taqman assay for RT PCR. The ddCT values demonstrated that the gene expression of IL- 6 and IL-8 was enhanced compared to a reference gene hPPIA. Similar results were obtained in a study of BM MSCs, with ddCT values of IL-6 showing similar enhanced expression [17]. In the study by Sutton et al., however, IL- 8 expression was downregulated by hMSC exposure, an important difference to note between the sources of hMSCs. Further, differences between the BM hMSCs and HCT hMSCs should be noted in the expression of CCL20 [17]. These results indicate that source variability in hMSCs should be taken into account on a disease specific basis. In a scenario such as $\mathrm{CF}$, where an acute neutrophil influx, indicated by high IL-8 expression, would be detrimental due to effects on neutrophil elastase, BM hMSCs may be the better alternative.
To assess the functionality of hMSCs, airway epithelial cells (A549) were treated with LPS and HCT hMSC. The A549 showed an increase in the production of IL- 6 and IL- 8 , following the trend seen in the HCT hMSCs alone, and again suggesting a disease-specific approach to selecting cell sources. However, another avenue to approach this variability in inflammatory marker expression between cell sources is to alter how the cells are grown. In order to study the possibility of inflammatory marker up or down regulation by altering growth culture medium, studies of HG and LG DMEM and Mes were assessed for optimization potential. The studies did not show overly significant differences in IL-6 expression with specific media overall. However, it should be noted that an increase in IL-8 expression using HG media occurred, suggesting LG or Mes media may be the best alternative in a scenario were a down-regulation of inflammation is called for.

HCT derived hMSCs could be a valuable source of hMSCs, especially with their high expression of IL-6 and other antiinflammatory markers. HCT hMSCs constitutively secrete cytokines, which are enhanced in the presence of bacterial analogs, demonstrating that hMSCs respond to and play an active role in the modification of their environment. The cellular activity assays confirmed that HCT hMSCs are active contributors to their environment and alter cytokine secretion. This cytokine secretion was also seen in airway epithelial cells, with trends towards antiinflammatory modulation through IL-6 expression. The antiinflammatory effect of HCT hMSCs may be able to be improved or tailored for disease specific therapeutic use under certain growth conditions, suggesting further pursuit of HCT hMSC growth optimization.

\section{Conclusion}

HCT hMSCs showed increased expression of IL-6 and IL-8 cytokines, as well as decreased CCL20 and increased SCF and MCP-1 expression, suggesting an overall trend towards an anti-inflammatory profile. HCT hMSCs secrete similar levels of IL- 6 and IL-8 to their cell based gene expression, as well as other anti-inflammatory factors including MIP1- $\alpha$, TNF- $\alpha$ and IL1- $\beta$. HCT hMSCs' ability to impact A549 IL-6 and IL-8 expression showed similar trends to the HCT hMSCs themselves, with heightened expression of both chemokines, both in the presence and absence of bacterial analogs. The inflammatory profile of HCT hMSCs may be altered by growth conditions, suggesting further arenas for optimization research for disease-specific therapeutic development [21-26].

As can be demonstrated by some of the larger margins of error, donor variability may factor into the results presented, suggesting the necessity for further donors to be assessed for a better understanding of the trends presented. Donor variability will also need to be refined further to determine the threshold quantity of hMSCs to be utilized to get a consistent and standardized response from patients, but also to determine or put into place screenings for HCT hMSC capabilities for optimal therapeutic benefit [27-33]. The obvious next steps of this study are to correlate optimal anti-microbial and anti-inflammatory potency as defined by our assays to specific mechanisms related to HCT hMSC impact on the individual immune cell targets outlined in our manuscript. Harnessing this information will provide important insight towards personalized optimization strategies for HCT hMSC therapeutics.

Future directions for this project will include testing the bacterial growth rate of bacterial species known to cause tissue damage in $\mathrm{CF}$ 
and other fatal pulmonary diseases, such as Pseudomonas aeruginosa and Staphylococcus aureus. This will allow a comparison of the effect of hMSCs on different bacteria. For a deeper, disease-specific analysis, study of the interaction between hMSCs with CF patient cells and preclinical models using CF cell lines will be conducted [34,35]. The final test of the efficacy of hMSCs is ultimately delivery to human subjects via the intravenous route or via inhalation to test their effectiveness in decreasing the inflammation and infection in chronic lung diseases. Numerous umbilical cord blood banks have established cord tissue storage programs in anticipation of future potential therapeutic applications of cord tissue derived cells. As has been noted by others, cord blood banks have the required infrastructure in place for donor screening and bio-banking and are familiar with applicable regulatory requirements [36]. Given the practical advantages that cryopreserved cord tissue presents as a source material, further understanding of the therapeutic potential of cord tissue hMSCs in chronic pulmonary diseases is warranted, and may ultimately lead to therapeutic benefit for millions suffering from debilitating disease in the world today.

\section{Acknowledgment and Funding}

We would like to thank the Case Western Reserve University Bioanalyte Core, Lung Modeling Core and the staff of Dr. Bonfield's laboratory, including but not limited to David Fletcher, Kelly Sopko, Vaishnavi Ragavapuram, Christiaan vanHeeckeren, and Lauren Auster for the insight and direction. We would also like to recognize the National Center for Regenerative Medicine for providing funding through the ENGAGE Summer Research Scholars Program, as well as Hathaway Brown School for their support through the Science Research and Engineering Program. Funding for these studies was generously provided through CBR Systems, Inc., South San Francisco, California.

\section{Disclosures of Interest}

CBR Systems, Inc. (Cord Blood Registry) is a wholly owned subsidiary of AMAG Pharmaceuticals, Inc. K. Brown, M. Skiles and H. Brown receive payment as employees of Cord Blood Registry and are stockholders in AMAG Pharmaceuticals.

\section{References}

1. Fathima M, Peiris D, Naik-Panvelkar P, Saini B, Armour CL (2014) Effectiveness of computerized clinical decision support systems for asthma and chronic obstructive pulmonary disease in primary care: a systematic review. BMC Pulm Med 14: 189-204. [PubMed]

2. Simpson G (2010) Investigation in chronic lung disease too much, too little, just right! Aust Fam Physician 39: 94-99. [PubMed]

3. Sutton MT, Fletcher D, Episalla N, Auster L, Kaur S, et al. (2017) Mesenchymal stem cell soluble mediators and cystic fibrosis. J Stem Cell Res Ther 7: 400. [PubMed]

4. Bonfield TL, Lennon D, Ghosh SK, DiMarino AM, Weinberg A, et al (2013) Cell based therapy aides in infection and inflammation resolution in the murine model of cystic fibrosis lung disease. Stem Cell Discovery 3 : 139-153.

5. Bonfield TL, Koloze M, Lennon DP, Zuchowski B, Yang SE, et al. (2010) Human mesenchymal stem cells suppress chronic airway inflammation in the murine ovalbumin asthma model. Am J Physiol Lung Cell Mol Physiol 299: 760-770. [PubMed]

6. Barry FP, Murphy JM (2004) Mesenchymal stem cells: clinical applications and biological characterization. Int J Biochem Cell Biol 36 568-584. [PubMed]
7. Watson N, Divers R, Kedar R, Mehindru A, Mehindru A, et al. (2015) Discarded wharton's jelly of the human umbilical cord: A viable source for mesenchymal stem cells. Cytotherapy 17: 18-24. [PubMed]

8. Skiles ML, Brown KS, Tatz W, Swingle K, Brown HL (2018) Quantitative analysis of composite umbilical cord tissue health using a standardized explant approach and an assay of metabolic activity. Cytotherapy 20: 564-575. [PubMed]

9. MesenCult ${ }^{\mathrm{Ta}}$ Proliferation Kit (2017) Stem Cell, Stem Cell Technologies, Inc. Accessed on: June 01, 2017.

10. Lennon DP, Caplan AI (2006) Isolation of human marrow-derived mesenchymal stem cells. Exp Hematol 34: 1604-1605. [PubMed]

11. Caimi PF, Reese J, Lee Z, Lazarus HM (2010) Emerging therapeutic approaches for multipotent mesenchymal stromal cells. Curr Opin Hematol 17: 505-513. [PubMed]

12. A549 (ATCC ${ }^{\oplus}$ CCL-185 ${ }^{\text {mat }}$ ) (2016) Essentials of Life Science Research, ATCC. Accessed on: February 01, 2016.

13. Pen Strep (2017) Penicillin Streptomycin, Thermo Fisher Scientific. Accessed on: August 01, 2017.

14. Bonfield TL, John N, Malur A, Barna BP, Culver DA, et al. (2004) Elevated monocyte chemotactic proteins 1,2 and 3 in pulmonary alveolar proteins are associated with chemokine receptor suppression. Clin Immunol 114: 79-85. [PubMed]

15. Bonfield TL, John N, Barna BP, Kavuru MS, Thomassen MJ, et al. (2005) Multiplexed particle-based anti-granulocyte macrophage colony stimulating factor (GM-CSF) assay used as pulmonary diagnostic test. Clin Diagn Lab Immunol 12: 821-824. [PubMed]

16. Luminex Assay Principle. Bio-Techne Corporation, R\&D Systems, 2017. Accessed on: March 01, 2017.

17. Sutton MT, Fletcher D, Ghosh SK, Weinberg A, van Heeckeren R, et al. (2016) Antimicrobial properties of mesenchymal stem cells: Therapeutic potential for cystic fibrosis infection, and treatment. Stem Cells Int 2016: 1-12. [PubMed]

18. Bonfield TL, Nolan MT, Lennon DP, Caplan AI (2010) Defining human mesenchymal stem cell efficacy in vivo. J Inflamm 7: 51-63. [PubMed]

19. Kumagai S, Ishida T, Tachibana H, Ito Y, Ito A, et al. (2015) Impact of bacterial coinfection on clinical outcomes in Pneumococcal pneumonia. Eur J Clin Microbiol Infect Dis 34: 1839-1847.

20. Caplan AI, Dennis JE (2006) Mesenchymal stem cells as trophic mediators. J Cell Biochem 98: 1076-1084. [PubMed]

21. Abumaree $\mathrm{MH}, \mathrm{Al}$ Jumah MA, Kalionis $\mathrm{B}$, Jawdat $\mathrm{D}, \mathrm{Al}$ Khaldi A, et al. (2013) Human placental mesenchymal stem cells (pMSCs) play a role as immune suppressive cells by shifting macrophage differentiation from inflammatory M1 to anti-inflammatory M2 macrophages. Stem Cell Rev 9: 620-641. [PubMed]

22. Archer NK, Mazaitis MJ, Costerton JW, Leid JG, Powers ME, et al. (2011) Staphylococcus aureus biofilms: properties, regulation, and roles in human disease. Virulence 2: 445-459. [PubMed]

23. ATCC Build 1.1.54.0. ATCC (2016) Penicillin-Streptomycin Solution $\left(\right.$ ATCC $^{\oplus} 30-2300^{\text {mit }}$ ). Essentials of Life Science Research, ATCC. Accessed on: March 03, 2016.

24. Brodsky JL, Frizzell RA (2015) A combination therapy for cystic fibrosis. Cell 163: 17. [PubMed]

25. Cash HA, Woods DE, McCullough B, Johanson WG Jr, Bass JA (1979) A rat model of chronic respiratory infection with Pseudomonas aeruginosa. Am Rev Respir Dis 119: 453-459. [PubMed]

26. DMEM, high glucose (2016) Thermo Fisher Scientific. Accessed on: June 01, 2017.

27. "Dulbecco's Modified Eagle's Medium (DMEM) (ATCC ${ }^{\otimes} 30-2002^{\mathrm{mm}}$ )." ATCC, ATCC Build 1.1.54.0. Accessed on: March 06, 2016.

28. HEPES. Millipore-Sigma, Sigma-Aldrich Co., (2017) Accessed on: August $01,2017$.

29. Human mag luminex performance assay base kit, HS Cytokine A. R\&D Systems Inc., Bio-techne. Accessed on: 06 March, 2016. 
Citation: Sutton MT, Kaur S, Brown KS, Skiles ML, Folz MA, et al. (2018) Anti-Inflammatory Therapeutic Development and Optimization of Umbilical Cord Tissue Derived Mesenchymal Stem Cells. J Stem Cell Res Ther 8: 435. doi:10.4172/2157-7633.1000435

Page 9 of 9

30. Kube D, Sontich U, Fletcher D, Davis PB (2001) Pro-inflammatory cytokine responses to P. aeruginosa infection in human airway epithelia cell lines. Am J Physiol Lung Cell Mol Physiol 280: 493-502. [PubMed]

31. Ledde O, Haller W, Couper RT, Lewindon P, Oliver M (2014) Cystic fibrosis: An update for clinicians. Part 2: Hepatobiliary and pancreatic manifestations. J Gastroenterol Hepatol 29: 1954-1962. [PubMed]

32. Lynch JP, Sayah DM, Belperio JA, Weigt SS (2015) Lung transplantation for cystic fibrosis: results, indications, complications, and controversies. Semin Respir Crit Care Med 36: 299-320. [PubMed]

33. Ong T, Ramsey BW (2015) Update in cystic fibrosis 2014. Am J Respir Crit Care Med 192: 669-675. [PubMed]
34. Heeckeren A, Walenga R, Konstan MW, Bonfield T, Davis PB, et al. (1997) Excessive inflammatory response of cystic fibrosis mice to bronchopulmonary infection with Pseudomonas aeruginosa. J Clin Invest 100: 2810-2815. [PubMed]

35. Zhang JM, An J (2007) Cytokines, inflammation and pain. Int Anesthesiol Clin 45: 27-37. [PubMed]

36. Zhou H, Rao MS (2015) Can cord blood banks transform into induced pluripotent stem cell banks? Cytotherapy 17: 756-764. [PubMed] 